\title{
Simulation on DC Current Distribution in AC Power Grid under HVDC Ground-Return-Mode
}

\author{
Guihua Mei ${ }^{1,2}$, Yuanzhang Sun', Yancun Liu ${ }^{2}$ \\ ${ }^{1}$ Department of Electrical Engineering, Tsinghua University, Beijing, China; ${ }^{2}$ Department of Electrical Power System Technique, \\ Guang Dong Electric Power Research Institute, GuangZhou, China. \\ Email: \{meiguihua, liuyancun\}@gddky.csg.cn, yzsun@mail.tsinghua.edu.cn
}

Received February 20 ${ }^{\text {th }}, 2010$; revised April 16 ${ }^{\text {th }}, 2010$; accepted April 25 ${ }^{\text {th }}, 2010$.

\begin{abstract}
This paper focus on the Modeling and Calculation of DC current distribution in AC power grid induced under HVDC Ground-Return-Mode. Applying complex image method and boundary element method, a new field-circuit coupling model was set up. Based on the calculation result with complex image method, this paper derived the modification factor for induced earth potential from practical measurement, which increased the accuracy of calculation. The modification method is helpful for evaluation on the effect of means used for blocking the dc-bias current in transformer neutral and also useful for the forecast of the DC current distribution when the power grid is in different line connection mode. The DC distribution character in Guangdong power grid is shown and suggestion is proposed that the mitigation of dc-bias should start from those substations whose earth-potential is highest.
\end{abstract}

Keywords: HVDC, DC-Bias, DC Distribution, Ground-Return Current, Simulation

\section{Introduction}

As an emergency mode, it's inevitable that HVDC transmission system operated in Ground-Return Mode (GRM) occasionally. When HVDC operate in GRM, as a result of current injection into soil through grounding electrodes, part of the return current will flow through the coils of the transformer whose neutral connected to the earth, and it will lead to wave distortion, noise and overheating etc. Sometimes it will lead to the damages of transformer or/and capacitor bank, disturbing the normal operation of power grid.

For evaluation on the range and degree of the influence by the HVDC in GRM, it is necessary to calculate the dc current distribution in ac network, summarizing characteristics of dc current distribution and providing data for the application of dc current blocking devices (DCBD). It's also need to evaluate the effects after using DCBD for better location.

In the related topic, most researches are focus on the calculation and analysis of earth potential distribution induced by ground return current of HVDC before 2003. Article [1] described a methodology that allows suitable calculations of the electric field as well as the potentials and current densities due to current in the ground electrode of a HVDC system in any point of nonhomogeneous soils and air media. Paper [2] presen- ted a method for the theoretical evaluation of the soil surface potentials induced in ground return mode and giving rise to soil models amenable to mathematical analysis. Paper [3] presented a method to evaluate the electric voltage and the current density in the immediate vicinity of a toroidal grounding installation of DC substations.

After 2005, related researches [4-14] report a lot.

2006, Dr Zhang Bo [4] used numerical methods to calculate the DC current distribution in AC power system caused by a HVDC system. Moment method is applied to calculate the electric fields in complex earth structure caused by all the grounding systems including DC grounding electrodes, AC substation grounding systems, and the long metal pipe lines. The circuit equations are coupled to the moment method to combine the AC transmission lines with the grounding systems.

Based on specified model, [5] and [10] calculated DC currents through earthed neutral transformers by using field circuit coupling method and the resistance network algorithm. It concluded that the resistance network algorithm can be applied to engineering instead of the using of field-circuit coupling method.

Paper [9] derived the Green's function of horizontalvertical-composite structure soil. The DC potential and current distribution caused by HVDC transmission's ground return mode was computed and DC current 
through transformers' earthed neutral under different soil structure was analyzed. Based on the physical meaning of Green's function, [6] solved the potential expression for horizontal-vertical-composite structure soil by applying the image method. In the same year, [8] studied DC bias problem of transformers of nuclear power plant and the character of DC current distribution, potential distribution of vertical grounding electrode and the DC capabilities of transformers were also carried out.

In the research report [7] by China Southern grid technology center and Tsinghua University, the fieldcircuit model was brought forward. This model considered inhomogeneity of soil. It was divided into two parts: the grounding network and the transmission line network. Applying this model, DC current distribution in AC power grid under HVDC transmission's ground return mode was analyzed. DC bias simulation model for three-phase compact transformer, also three-phase and three-limb transformer, three-phase transformer with five-limb core, is presented. The analyzed method for 3 dimensions for eddy field is put forward. The influence of HVDC transmission's ground return current to transformers is analyzed. Some rules about the difference of different structure transformer and mitigation transformer's DC bias were proposed.

Although lots of researches have been made, there are still many questions to be investigated.

- The diversity and complexity of geological condition leads to low accuracy of simulation and the simulation result will be unauthentic.

- The simulation result should be checked out by lots of measuring data.

Theoretical and practical research of the $220 \mathrm{kV}$ and $500 \mathrm{kV}$ substations in Guangdong power grid was carried out on the base of the online measuring system of DC current through transformers' neutral. Further research of modification for the theoretical model by the data of measuring system was accomplished.

\section{Calculation Model}

The model for power network above ground was described by electrical circuit method while the model for underground part was described by the field model which was shown in the electrical circuit way. By these models, we combine the circuit network above ground with the field effect underground to solve the problem.

The underground model of substation, which is expressed by a DC ground resistance in series with an earth potential source, is shown in Figure 1.

$R$ is the equivalent resistance between the transformer's neutral and the zero-potential point (the farthest point).

$P$ is the earth potential induced by HVDC ground return current and the grounded current of other substation.
In this paper, the solution method for $P$ was complex image method or boundary element method on the assumption that $P$ are related with the HVDC ground return current, the position of the substation and DC grounding electrode, geological condition, but independent of operation mode of power grid.

The whole model of dc distribution was shown in Figure 2.

By nodal analysis of circuit theory, we have

$$
G V=S P
$$

In (1), $G$ is the conductance matrix, $V$ is the nodal voltage matrix, $P$ is the earth potential of substation, $S$ is the transfer matrix of earth potential and nodal current.

The relationship between nodal current and earth potential was shown as follow:

$$
P=Y I+M I_{D C}
$$

In (2), $Y$ is the mutual resistance matrix between substations, $I$ is the injected current matrix of substations, $M$

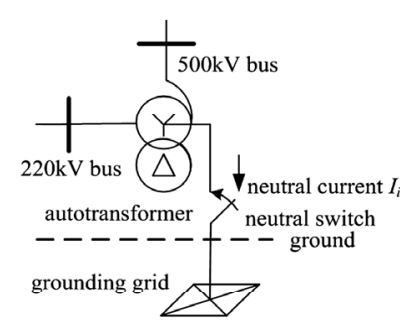

(a) A $500 \mathrm{kV}$ substation

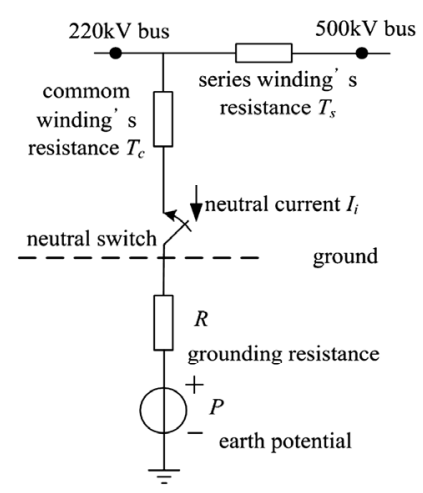

(b) The field-circuit coupling model
Figure 1. The network model of substation

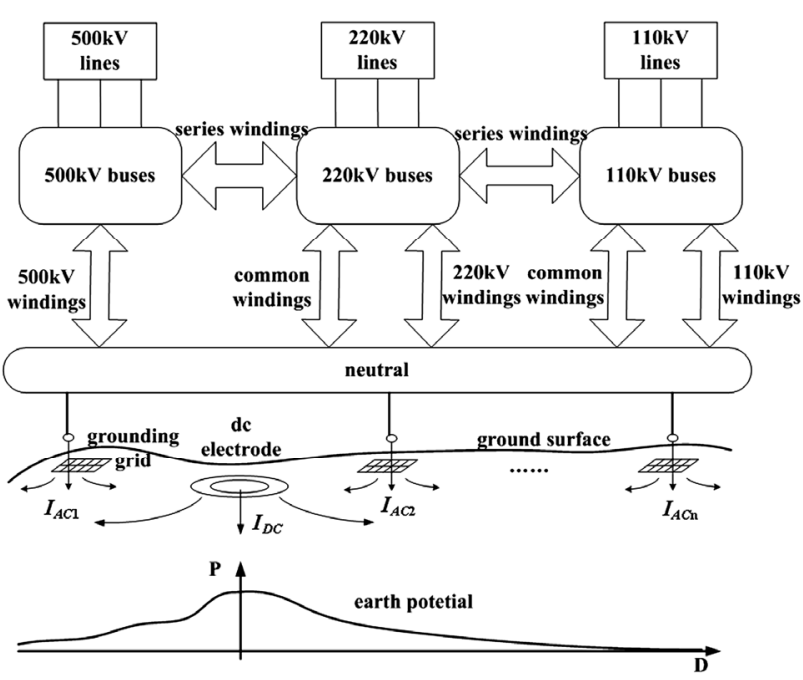

Figure 2. The whole model of dc distribution 
is the mutual resistance matrix between substations and dc electrode, $I_{D C}$ is the injected current of dc electrode.

The relationship between nodal voltage and injected current was shown as follow:

$$
I=X V
$$

In (3), $X$ is the transfer matrix of nodal voltage and injected current.

From (1)-(3), we have

$$
(G-S Y X) V=S M I_{D C}
$$

Equation (4) shows injected current drives $D C$ distribution in ac power grid and dc distribution is decided by the $D C$ network parameters.

\section{Comparison between Complex Image Method and Boundary Element Method}

Complex image method (CIM) and boundary element method (BEM) are of different characters when applied in the large scale grounding problem.

CIM is relatively simple. mountain, river and ocean are not took into account, only the equivalent horizontal stratified structure soil is needed, regardless of the subdivision of boundary element. The program is relatively simple, the consumption of CPU time and PC memory are much less than BEM.

In theory, BEM is the most proper method for the complexity and difference of geological condition. But subdivision of boundary element, also the coupling of different elements is needed. When the number of boundary element becomes large, CPU time and memory are greatly consumed. There are some questions when large scale BEM program operated in personal computer. By example of CDEGS of SES Corporation, the upper limit for the number of boundary element is about 20000 when the software used in PC. For the problem in this paper, 20000 boundary elements can not fully expressed the complexity and difference of geological condition in $\mathrm{Gu}-$ angdong (the area of Guangdong is about $180000 \mathrm{~km}^{2}$ ).

XinAn HVDC system was taken as example for comparison of the two methods. By validation of measuring data, we will find the better method to solve this problem.

\section{Complex Image Model}

The horizontal stratified structure soil is shown in Figure 3. $h$ is the thickness of the soil while $\rho$ is the resistivity, and $I$ is the current point.

Applying Prony method [15], the Green's function of horizontal stratified structure soil is:

$$
\begin{aligned}
& \phi(r, z)=\frac{\rho I}{4 \pi} \frac{1}{\sqrt{r^{2}+z^{2}}} \\
& +\frac{\rho I}{4 \pi} \sum_{i=1}^{M}\left[\frac{a_{i}}{\sqrt{r^{2}+\left(z-b_{i}\right)^{2}}}+\frac{c_{i}}{\sqrt{r^{2}+\left(z-d_{i}\right)^{2}}}\right]
\end{aligned}
$$

where: $a_{i}$ and $c_{i}$ is the amplitude of complex image, $b_{i}$ and $d_{i}$ is the position of complex image.

For approximation, all the $\mathrm{DC}$ and $\mathrm{AC}$ grounding electrodes were viewed as point source. So the earth potential of substation induced by current point source could be got by (1).

The earth potential induced by XinAn HVDC ground return current with $600 \mathrm{~A}$ could be obtained and the earth potential curve is shown in Figure 4. The soil parameter is listed in Table 1.

Figure 4 shows that earth potential decrease rapidly while $d<20 \mathrm{~km}$. Potential difference between line-connected substations may lead to dc bias of transformers.

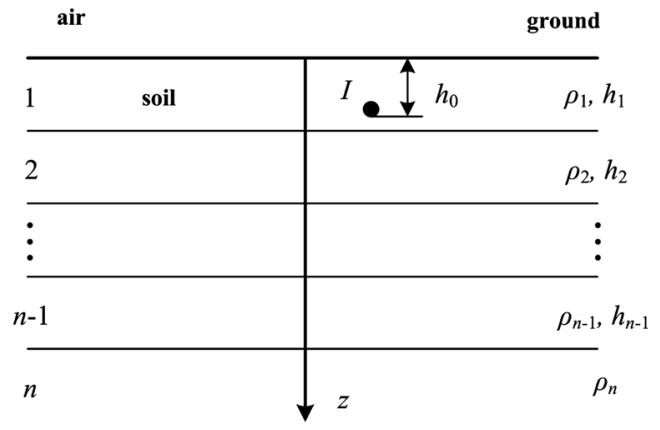

Figure 3. Model of multi-layered soil

Table 1. 4-layer horizontal stratified soil parameter

\begin{tabular}{ccc}
\hline layer number & Resistivity $(\Omega \cdot \mathrm{m})$ & thickness $(\mathrm{m})$ \\
\hline 1 & 235 & 30 \\
2 & 5900 & 1000 \\
3 & 14100 & 50000 \\
4 & 120 & inf \\
\hline
\end{tabular}
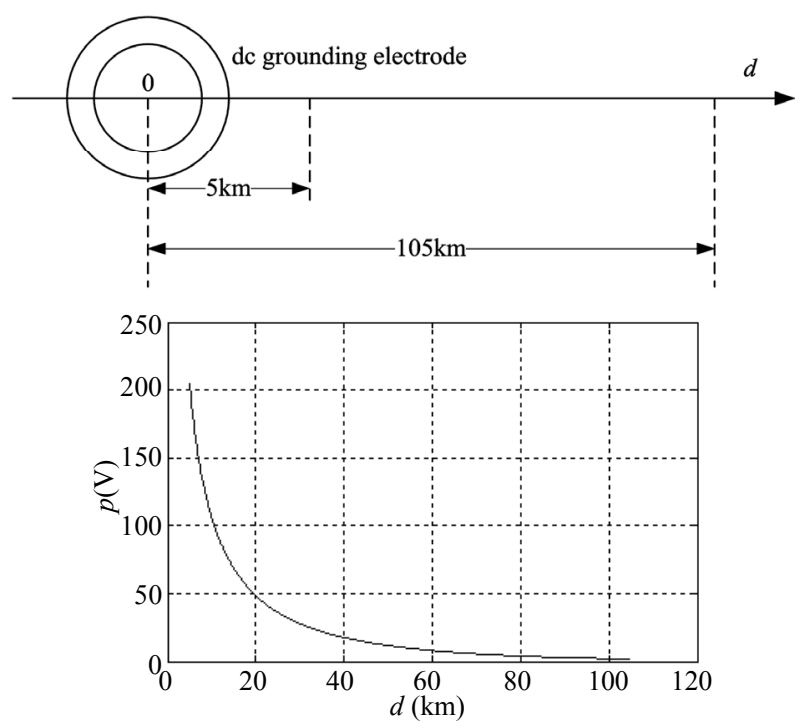

Figure 4. Earth potential 


\section{Method of Model Modification}

Because of the complexity and difference of geological condition, the approximation will bring down the veracity of the model and even the simulation result will become invalid.

This paper presented a feasible method to overcome this shortage. Applying the measuring data, provided by the online monitoring system of DC current through the neutral of transformer, we modify the parameter of the model.

The core of this method is to modify the earth potential $P$ by the measuring data. The question of modifying the earth potential could be solved by the least-square method.

Supposed the injecting current of DC electrode is $x$. there are $m$ substations' earth potentials to be modified. Let

$$
r_{i}(x)=I_{i}(x)-J_{i}(x), i=1, \cdots m
$$

where $I_{i}(x)$ are the measuring data, $J_{i}(x)$ are the computational result by CIM.

$$
f(x)=\frac{1}{2} \sum_{i=1}^{m} r_{i}^{2}(x), x \in R^{n}, m \geq n
$$

Let

$$
r(x)=\left(r_{1}(x), r_{2}(x), \cdots, r_{m}(x)\right)^{T}
$$

The objective function is:

$$
f(x)=\frac{1}{2} r(x)^{T} r(x)
$$

This equation could be solved by numerical analysis of Gauss-Newton.

In many practical problems, if $x^{*}$ is the locally solution and corresponding objective function value $f\left(x^{*}\right)$ close to 0 , when the iterative point close to $x^{*}$, or the curve $r_{i}(x)$ is almost a straight line $\left(\nabla^{2} r_{i}(x) \approx 0\right)$, the Gauss-Newton method can produce a good effect. But when $\left\|r\left(x^{(k)}\right)\right\|$ is large, or $r_{i}(x)$ 's curvature is large, $\nabla^{2} f\left(x^{(k)}\right)$ ignores $\sum_{i=1}^{m} r_{i}(x) \nabla^{2} r_{i}(x)$ which is not neglectable, so the Gauss-Newton method can't get the right answer.

Obviously, $J\left(x^{(k)}\right)^{T} J\left(x^{(k)}\right)$ is a Positive semidefinite matrix. If Jacobi matrix $J\left(x^{(k)}\right)$ is column full rank, matrix $J\left(x^{(k)}\right)^{T} J\left(x^{(k)}\right)$ is positive definite matrix. So $d^{(k)}$, getting from the equations, is agree with the descending direction of $f(x)$, but $f\left(x^{(k+1)}\right)<f\left(x^{(k)}\right)$ isn't confirmed, then additional one-dimension search is needed as the Newton method.

\section{Examples}

XinAn HVDC system consists of a $1200 \mathrm{~km}$ long bipolar $\pm 500 \mathrm{kV}_{\mathrm{dc}}$ transmission line with transmitting capacity of 3000MW in Southern China. When HVDC system operates in ground return mode, 3000 A direct current will inject into the earth.

Under the transmission lines connection of Guangdong power grid in Apr 2007 and suppose that $600 \mathrm{~A}_{\mathrm{DC}}$ current injected into the earth by HVDC, The result of transformer neutral dc current at some substation by CIM and BEM was listed in Table 2. The geological condition was approximate to horizontal 4 layers soil for complex image method. The BEM boundary condition was determined by the geologic exploration result.

From Table 2, we can see,

1) The result of YingDe-PaJiang-ChongHua by CIM is close to measuring data, but BeiJiao's computation result was near 3 times of measuring data. As a whole, horizontal 4 layers soil is suitable for the local geological condition.

2) The difference between computation and measuring for QingYuang, KangLe, SangShui and LuoDong was large. The reason is another HVDC system (TianGuang) was then under bipolar operation mode with unbalanced power of $400 \mathrm{MW}$, which influence the earth potential of the substations on the path of QingYuan-KangLe-SangShui-LuoDong, when the measuring data were recorded. The event leads to the measuring data stand for not only the influence of XinAn HVDC system and the error. But however the correction of the method presented in this paper do not be influenced.

Although actual geological condition of Guangdong province was considered and LAPACK for matrix algorithm is used, problems were still found that BEM demands huge CPU time and occupied big size memory.

Table 2. Computational result and measuring data

\begin{tabular}{cccc}
\hline Name & $\begin{array}{c}\text { Complex Image } \\
\text { Method (A) }\end{array}$ & $\begin{array}{c}\text { Measuring } \\
\text { Data (A) }\end{array}$ & $\begin{array}{c}\text { Boundary } \\
\text { Element Method (A) }\end{array}$ \\
\hline PaJiang & 25.7 & 25.9 & 8.25 \\
YingDe \# 1T & 11.5 & 12.0 & 3.90 \\
ChongHua & 12.2 & 12.9 & 3.51 \\
SanShui & 17.8 & 3.0 & 25.52 \\
KangLe & 33.8 & 7.5 & 37.23 \\
BeiJiao & 17.5 & 6.0 & 23.08 \\
LuoDong & 1.1 & 9.0 & 1.62 \\
QingYuan & 21.4 & 3.0 & 33.77 \\
\hline
\end{tabular}


Boundary element's number $\mathrm{N}$ is limited as computing time grows proportionally to $N^{3}$, which leads to the boundary division being rough and the geological condition could not be simulated properly, especially for rivers and ocean. Another side, the lacking of geological condition parameter in large area for DC current conducting also leads to simulation error.

\subsection{Example for the Method of Model Modification}

Based on the operation mode of Guangdong power grid in Apr 2007, the modification model for complex image method was used to solve DC distribution when XinAn HVDC system was under commissioning in 2007. Comparison between computation result and measuring data is shown in Table 3.

From the result above we could see that in large area, the simulation result of the transformer neutral dc current are more close to the measuring data without appearance of big error. The modification factor getting by the field measuring data will be helpful for these simulations, such as changing of transmission line connection, being built of new transmission line or the effect evaluation of DCBD using, etc.

\section{Summary on the DC Distribution in Guangdong Power Grid}

According to the operation mode of Guangdong power grid in the first half of 2008, the correction model was used to analyze the DC bias caused by the 4 HVDC transmission projects in Guangdong power grid. Some conclusions are listed as follows.

When HVDC system is under ground return mode, the transformer, whose location is close to the location of electrode of HVDC system, is easier suffering from DC bias. The transformers in the transmission line path, which connect the substation with higher earth potential and the substation with lower earth potential, might also suffer from DC bias. Generally the substations far away from the electrode location, especially those that close to river or ocean are of the lower earth potential. The electrode

Table 3. Comparison between computation result and measuring data when XiAn HVDC system operated in GRM (600ADC current injected into the earth)

\begin{tabular}{cccc}
\hline Name & Modification factor & Current (A) & Measuring data (A) \\
\hline QingYuan & 0.63 & 0.58 & 3.0 \\
KangLe & 1.93 & 7.50 & 7.5 \\
SanShui & 2.16 & 2.41 & 3.0 \\
XianXi & 1.10 & 2.26 & 1.0 \\
LuoDong & 2.27 & 14.56 & 9.0 \\
PaJiang & 0.92 & 27.13 & 25.9 \\
YingDe & 0.95 & 7.92 & 12.0 \\
ChongHua & 0.90 & 13.17 & 12.9 \\
BeiJiao & 0.67 & 5.35 & 6.0 \\
\hline
\end{tabular}

locations of the four HVDC projects in Guangdong province are illustrated in Figure 5.

For TianGuang HVDC system, LuoDong, SanShui, XianXi, GuoTang are close to the DC grounding electrode of TianGuang HVDC system. Because of the high amplitude earth potential of the above substations, the DC current through the transformers' neutral are relative big by the monitoring data. Meanwhile the high earth potential at those substations leads to DC current goes through the transmission line to other substations with lower DC earth potential. Most of the transformers, which connected with the transmission lines of SanShui $\rightarrow$ KangLe $\rightarrow$ QingYuan or LuoDong $\rightarrow$ HongXing $\rightarrow$ ZiDong $\rightarrow$ XiJiang $\rightarrow$ FenJiang, are in DC bias. Another typical line is LuoDong $\rightarrow$ XiJiang $\rightarrow$ JiangMen $\rightarrow$ KaiPing $\rightarrow$ TaiShan $\rightarrow$ TangMei, which begins from the substation near the HVDC grounding electrode and end at the substation near the coast of South China Sea. Especially TangMei near the coast suffered severe DC bias for the DC earth potential close to coast is near to zero.

There are $4 \pm 500 \mathrm{kV}$ HVDC systems which transmit power from western China to Guangdong power grid in 2008. When different HVDC system is in ground return mode, there is similar rule for the transformers which might be in dc-bias by the result of simulation and monitoring measures.

From the rule of DC current distribution, we could find that the substations with higher induced dc earth potential should be installed the DCBD first when mitigation of dc-bias is under schedule.

\section{Conclusions}

1) Comparison between complex image method and BEM shows BEM isn't suitable for the solution of grounding problem in large scale complex geological condition for the limits of PC hardware. Although complex image method couldn't take geological condition into account completely, proper choice for the soil structure and parameter still could simulate the local geological condition precisely.

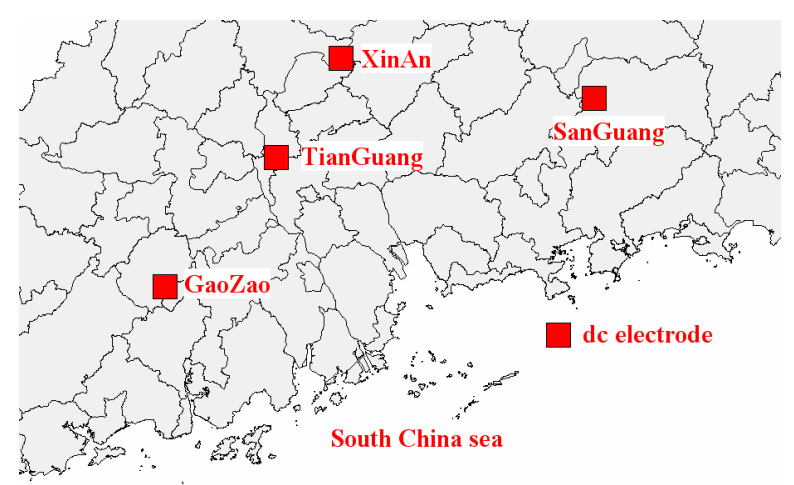

Figure 5. DC grounding electrodes in Guangdong 
2) Utilizing the measuring data, the correction model was presented. This method is significant for the accuracy improvement of simulations, such as changing of transmission line connection, or the effect evaluation of DCBD installation, etc.

3) When HVDC system is under ground return mode, the transformer, whose location is close to the ground electrode of HVDC system, is easier suffering from DC bias. Another case, the transformers which located far away from the ground electrode of HVDC system, with lower dc earth potential, especially those that close to river or ocean, and connected to the substation with higher dc earth potential might also easier suffer from DC bias.

4) The substations with higher induced dc earth potential should be installed the DCBD first when mitigation of dc-bias is under schedule.

\section{REFERENCES}

[1] E. T. V. Jose and M. P. Carlos, "Calculation of Electric Field and Potential Distributions into Soil and Air Media for a Ground Electrode of a HVDC System," IEEE Transactions on Power Delivery, Vol. 18, No. 3, 2003, pp. 867873.

[2] D. Kovarsky, L. J. Pinto, C. E. Caroli, et al., "Soil Surface Potentials Induced by ITAIPU HVDC Ground Return Current Part I-Theoretical Evaluation," IEEE Transactions on Power Delivery, Vol. 3, No. 3, 1988, pp. 12041210.

[3] P. J. Lagace, J. L. Houle, Y. Gervais, et al., "Evaluation of the Voltage Distribution around Toroidal HVDC Ground Electrodes in N-Layer Soils," IEEE Transactions on Power Delivery, Vol. 3, No. 4, 1988, pp. 1573-1579.

[4] B. Zhang, J. Zhao, R. Zeng, et al., "Estimation of DC Current Distribution in AC Power System Caused by HVDC Transmission System in Ground Return Status," Proceedings of the Chinese Society for Electrical Engineering, in Chinese, Vol. 26, No. 13, 2006, pp. 84-88.

[5] Z. J. Cao, J. J. He, H. S. Ye, et al., "Method for the Calculation of DC Current Distribution in AC System when HVDC Operating in Ground-Return Mode," High Voltage Engineering, in Chinese, Vol. 32, No. 10, 2006, pp. 82-84.

[6] Q. Liu, L. C. Li and J. C. Zheng, "DC Currents Distribu- tion in HVDC Systems of Monopolar Operation with Ground Return in Complex Soil Structure," Proceedings of the Chinese Society for Electrical Engineering, in Chinese, Vol. 27, No. 36, 2007, pp. 8-13.

[7] China Southern Grid Technology Center, "Influence to AC System and Other Facilities by Ground Return Currents of HVDC and the Study of Preventive measures," Project Research Report, Electrical Engineering Department of Tsinghua University, Beijing, 2005.

[8] Q. Liu, "Study on Ground Currents Distribution while HVDC Mono-Polar is Operating," Chinese Dissertation Abstracts Database, Tsinghua University, Beijing, 2007.

[9] J. J. He, H. S. Ye, F. C. Lin and H. Li, "HVDC Influence of Soil Structure on Surface Potential and Ground Currents Distribution while HVDC Mono-Polar Operation with Ground Return," Southern Power System Technology, Vol. 1, No. 1, 2007, pp. 20-36.

[10] H. S. He, "Computational Method Study on DC Current Distribution in AC System when HVDC Operating in Ground-Return Mode. Power Technology Development and Energy Conservation," The 9th Symposium on Youth Essays in Chinese Society for Electrical Engineering, November 2006.

[11] Z. Q. Ma, X. L. Li and D. Z. Zhang, "Network Algorithm to Analyze Effects of DC Transmission Ground Current on AC System," Guangdong Electric Power, in Chinese, Vol. 18, No. 12, 2005, pp. 4-8.

[12] Q. Liu, L. C. Li and J. C. Zhen, "Study on DC Current through Transformer Neutrals Caused by Ground Return Operation Mode of HVDC System with Sea Influence Considered," Power System Technology, in Chinese, Vol. 31, No. 2, 2007, pp. 57-60.

[13] J. Zhao and B. Zhang, "Analysis on Influence of Ground Electrode Current in HVDC on AC Power Network," Power System Technology, in Chinese, Vol. 29, No. 19, 2005, pp. 60-64.

[14] J. M. Lu, D. Xiao, C. X. Mao, et al., "Analysis of Effects of DC Earthed Pole on Earth Surface Potential Distributions," High Voltage Engineering, in Chinese, Vol. 32, No. 9, 2006, pp. 55-58.

[15] Y. L. Chow, "Complex Images for Electrostatic Field Computation in Multilayered Media," IEEE Transactions on Microwave Theory and Techniques, Vol. 39, No. 7, 1991, pp. 1120-1125. 